\title{
Neck Lymph Node Boundary Level III
}

National Cancer Institute

\section{Source}

National Cancer Institute. Neck Lymph Node Boundary Level III. NCI Thesaurus. Code C132499.

The following anatomical structures define the neck lymph node boundary for level III: Superior: horizontal plane defined by the inferior body of the hyoid; inferior: horizontal plane defined by the inferior border of the cricoid cartilage; anterior (medial): lateral border of the sternohyoid muscle; posterior (lateral): lateral border of the sternocleidomastoid or sensory branches of the cervical plexus. (AJCC 8th ed.) 